\title{
A Redox-Sensitive Micelle-Like Nanoparticle Self-Assembled from Amphiphilic Adriamycin-Human Serum Albumin Conjugates for Tumor Targeted Therapy
}

\author{
Lin Chen, ${ }^{1}$ Feng Chen, ${ }^{2}$ Mengxin Zhao, ${ }^{3}$ Xiandi Zhu, ${ }^{3}$ Changhong Ke, ${ }^{3}$ \\ Jiangming Yu, ${ }^{4}$ Zhiqiang Yan, ${ }^{5}$ Fulei Zhang, ${ }^{3}$ Yun Sun, ${ }^{3}$ Di Chen, ${ }^{3}$ Cheng Jiang, ${ }^{3}$ \\ Xianxian Zhao, ${ }^{2}$ Yong Gao, ${ }^{1}$ Shangjing Guo, ${ }^{3}$ and ${\mathrm{Wei} \mathrm{Li}^{3}}^{3}$ \\ ${ }^{1}$ Department of Medical Oncology, East Hospital, Tongji University, 150 Jimo Road, Shanghai 200120, China \\ ${ }^{2}$ Department of Cardiology, Changhai Hospital, The Second Military Medical University, Shanghai 200433, China \\ ${ }^{3}$ International Joint Cancer Institute, The Second Military Medical University, Shanghai 200433, China \\ ${ }^{4}$ Department of Orthopaedics, Changzheng Hospital, The Second Military Medical University, No. 415 Fengyang Road, \\ Shanghai 200003, China \\ ${ }^{5}$ Institute of Biomedical Engineering and Technology, Shanghai Engineering Research Center of Molecular Therapeutics, \\ Shanghai 200062, China
}

Correspondence should be addressed to Yong Gao; gaoyon@hotmail.com and Wei Li; liwei@smmu.edu.cn

Received 15 January 2015; Revised 26 March 2015; Accepted 7 April 2015

Academic Editor: Yunfeng Zhao

Copyright (C) 2015 Lin Chen et al. This is an open access article distributed under the Creative Commons Attribution License, which permits unrestricted use, distribution, and reproduction in any medium, provided the original work is properly cited.

The application of chemotherapeutic drug adriamycin (ADR) in cancer therapy is limited by its side effects like high toxicity and insolubility. Nanomedicine offers new hope for overcoming the shortcomings. But how to increase in vivo stability and to control intracellular drug release is a key issue for nano-based formulations. Herein, the hydrophobic ADR was successfully linked to the biocompatible human serum albumin (HSA) by disulfide bond 3-(2-pyridyldithio) propionyl hydrazide (PDPH), resulting in amphiphilic HSA-ADR. The novel ADR-HSA micellar NPs which were thus assembled exhibited a well-defined stable core shell structure with glutathione (GSH) sensitive linkers. The stable PDPH linkers at extracellular level were broken by GSH at intracellular level with a controlled ADR release profile. The in vitro cytotoxicity against gastric cancer cells (NCI-N87) was obviously enhanced by such redox-sensitive ADR-HSA NPs. Additionally, as observed by IVIS Lumina II Imaging System (Xenogen), the intratumor accumulation of ADR-HSA NPs was much higher than that of HSA/ADR NPs due to its high stability. Consequently, the in vivo tumor inhibition was significantly promoted after intravenous administration to the Balb/c nude mice bearing gastric tumors. These in vitro/vivo results indicated that disulfide-bond-containing ADR-HSA NPs were an effective nanodrug delivery system for cancer therapy.

\section{Introduction}

Adriamycin (ADR) is a kind of anthracyclines antitumor drug, which has strong cytotoxic effect and has been widely used in the treatment of liver cancer, lymphoma, gastric cancer, and breast cancer [1,2]. But its low aqueous solubility and serious side effects such as bone marrow suppression, immunosuppression, and cardiac toxicity limited its clinical application $[3,4]$. In order to reduce the side effects, nanoparticles such as liposomes, nanoparticles, and polymer micelles have been developed for improving the therapeutic effect $[5,6]$, among which, the new drug delivery system, HSA nanoparticles have received extensive attention due to its good biocompatibility, low toxicity and irritation, nonimmunogenicity, easy preparation, as well as the feasibility of drug targeting, sustained drug release and increased drug stability. HSA nanoparticles were regarded as a good drug carrier by fast accumulating in the rapid growing tumor tissues and delivering nutrition to cells with the intratumor drug release and improved the therapeutic effect $[7,8]$. 
The HSA based formulation that was the paclitaxel (PTX) albumin nanoparticles solution (ABRAXANE) developed by American bioscience has been approved by the Food and Drug Administration (FDA) for clinical cancer therapy [9]. Clinical results showed that the HSA/PTX formulation was a potent antitumor drug. However, being an anticancer drug carrier material, HSA based formulations still created some problems to be solved including the low serum stability, easily to be broken, broad size distribution, and low intratumor accumulation of drugs, which was attributed to deformation of the physical blends [10]. The stability could be enhanced if the drugs and the HSA are linked together by chemical crosslinkers. The drugs and HSA linked by covalent bond can selfassemble into the micellar drug delivery system with stable structure and high tumor uptake efficiency [11, 12]. Besides, HSA is an ideal material for conjugating chemotherapy drugs, since it is a nutrient in the process of tumor proliferation $[13,14]$. Therefore, the conjugation of antitumor drug (ADR) with HSA may result in reducing the systemic toxicity and increasing therapeutic effect of ADR.

It is known that the intracellular environment is different from extracellular environment which was characterized by the lower $\mathrm{pH}(4-6)$ and the redox states [15]. Intracellular glutathione (GSH/GSSG) concentration $(0.5 \sim 10 \mathrm{~mol} / \mathrm{L})$ is over 200 times the extracellular GSH concentration (2 $20 \mu \mathrm{mol} / \mathrm{L}$ ) [16]. The GSH in cells plays a crucial role for the redox condition regulation $[17,18]$. In addition, compared with normal tissue cells, the tumor tissue cells have a more reduced environment due to their hypoxic states $[19,20]$. All the above-mentioned concerning unique characteristics of intracellular conditions and tumor microenvironments offer new hope to screen a redox-sensitive bond for development of a reduction-sensitive drug carrier [21-23]. Among redoxsensitive bonds, the disulfide bond 3-(2-pyridyldithio) propionyl hydrazide (PDPH) is an ideal candidate. This PDPH can remain stable under the low concentration of GSH outside the cells. But it can be broken by the high concentration of GSH (intracellular level), resulting in the degradation of carrier, release of drug, and inhibition of tumor growth [24-28]. At present, the reduction sensitive bond is widely used in the drug/gene delivery system. And some new disulfide bonds were developed for controlled release recently [29].

In this study, the disulfide bond PDPH was utilized to link the ADR to the HSA, resulting in reduction-sensitive amphiphilic copolymer HSA-ADR. Then the novel micellarlike ADR-HSA nanoparticles were assembled by a rotary evaporation technique. The nanocarrier's properties, in vitro and in vivo antitumor effects were systemically investigated. This study provided an easy and feasible idea for the design and preparation of reduction-sensitive nanodrug delivery system.

\section{Materials and Methods}

2.1. Materials. Adriamycin was obtained from Dalian Meilun Biology Technology Co. 3-(2-Pyridyldithio) propionyl hydrazide (PDPH) was obtained from Pierce. HSA was obtained from Sigma with purity of 99\%. 2-Iminothiolane hydrochloride (2IT) was purchased from Sigma. Dulbecco's modified Eagle's medium (DMEM) and Fetal bovine serum (FBS) were purchased from Gibco Co., USA. All chemicals were in analytic reagent grades.

2.2. Cells and Animals. The human stomach cancer NCI-N87 cell line, which was purchased from Shanghai Institute of Cell Biology, was cultured in DMEM supplemented with 10\% FBS and $1 \%$ penicillin and streptomycin at $37^{\circ} \mathrm{C}$ with $5 \% \mathrm{CO}_{2}$.

Male Balb/c mice (4-6 weeks, 20 g) were obtained from Shanghai Experimental Animal Center of Chinese Academic of Sciences (Shanghai, China) and kept under SPF conditions. The animal experiments were performed in accordance with guidelines provided by the ethics committee of the Second Military Medical University, Shanghai, China.

\subsection{Synthesis and Characterization of ADR-HSA. ADR-HSA} was synthesized according to the procedure shown in Figure $1.2 \mathrm{~mL}$ adriamycin solution $(4 \mathrm{mg} / \mathrm{mL})$ was mixed with $2 \mathrm{mg} \mathrm{PDPH}$ and reacted in dark for a week. After reaction, the solution was dialyzed with a dialysis bag (MW cut off 3500) against dd water for 4 to $6 \mathrm{~h}$ to obtain the ADR-PDPH solution. The HSA was dissolved in dd water $(2 \mathrm{mg} / \mathrm{mL})$ and was thiolated through the reaction with 2IT. Then $2 \mathrm{~mL}$ ADR-PDPH solution $(2 \mathrm{mg} / \mathrm{mL})$ was mixed with $2 \mathrm{~mL}$ thiolated with molar ratio of ADR/HAS 50/1 and reacted for 2-3 h to obtain the ADR-HSA solution.

2.4. Preparation of ADR/HSA Nanoparticles (ADR/HSA NPs) and ADR-HSA Nanoparticles (ADR-HSA NPS). In the experiments, $0.5 \mathrm{mg}$ ADR-HSA was dissolved in $1.5 \mathrm{~mL}$ chloroform followed by drying under $\mathrm{N}_{2}$ atmosphere to form a thin film. Then the flask was connected with the equipment of rotation evaporation. The ADR-HSA film was eluted by $2 \mathrm{~mL}$ dd water; as a result, ADR/HSA NPs solution with concentration about $0.25 \mathrm{mg} / \mathrm{mL}$ was obtained. On the other hand, the ADR/HSA NPs solution were just prepared by mixing the drug ADR with HSA with the same concentration as ADR-HSA and dissolved in $2 \mathrm{~mL}$ dd water. For evaluation of cytotoxicity of the ADR-HSA nanoparticles (ADR-HSA NPs), the blend of the ADR/HSA nanoparticles (ADR/HSA NPs) at the same ADR concentration was served as control.

2.5. Size Distribution and Surface Potential of ADR-HSA NPs. The size distribution of ADR-HSA NPs was tested by the dynamic laser light scattering instrument (DLLS, ALV/CGS3 , Germany) at the scattering angle of $30^{\circ}$. The surface potential $(\zeta)$ of ADR/HSA and ADR-HSA NPs was tested by a Malvern Zetasizer 3000HS (Malvern, UK) using $5 \mathrm{mM} \mathrm{NaCl}$ as the baseline.

2.6. Transmission Electron Microscopy (TEM). The morphology of particles was characterized by TEM and AFM. To prepare stained specimens for TEM (Hitachi, H-7000 Electron Microscope), PL-RB solution $(0.5 \mathrm{mg} / \mathrm{mL})$ was dropped on a 200-mesh Formvar-free carbon-coated copper grid (Ted Pella Type-A). After water evaporated, the sample was inversely 
<smiles>COc1cccc2c1C(=O)c1c(O)c3c(c(O)c1C2=O)C[C@@](O)([C@H](O)CO)C[C@H]3C[C@H]1C[C@H](N)[C@@H](O)[C@H](C)O1</smiles>

\section{DDDODODDODODOR- $\mathrm{NH}_{2}$}

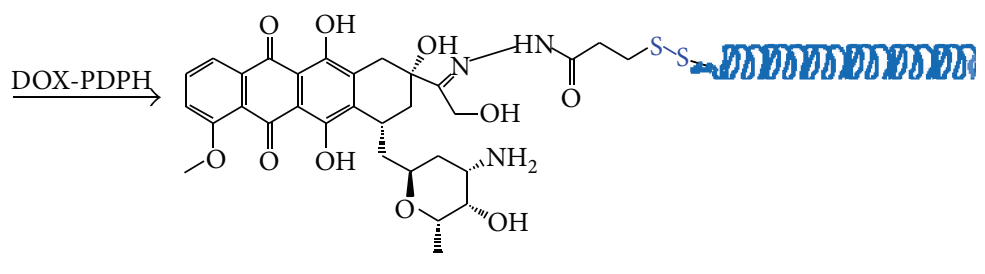

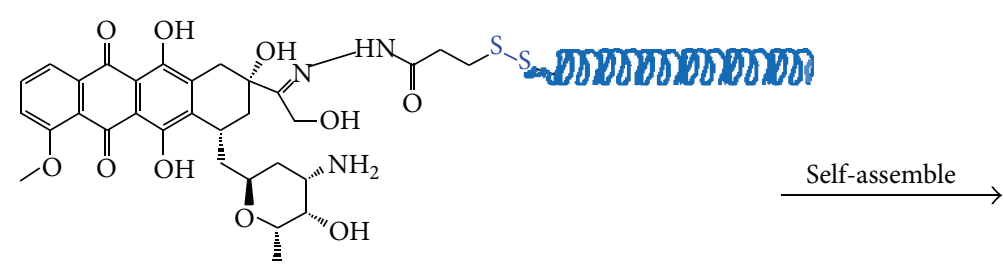

HSA-DOX

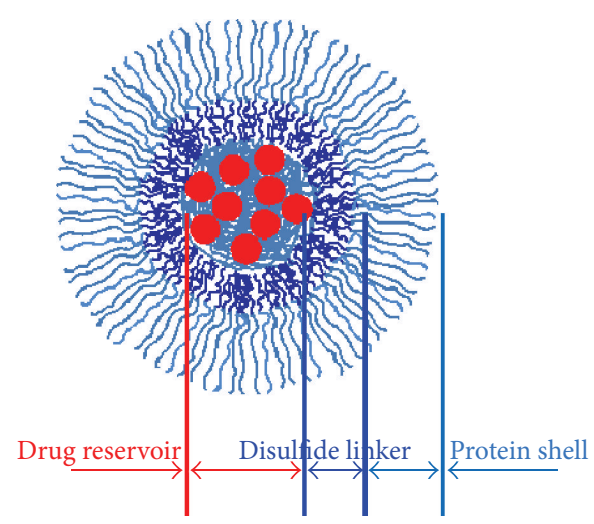

FIGURE 1: The scheme illustrated the synthesis and assembly process of ADR-HSA nanoparticles with well-defined structure.

covered on a small drop of $2 \%$ hydrodated phosphotungstate (PTA) solution. The conventional TEM images were obtained at $100 \mathrm{kV}$.

2.7. The In Vitro Release of ADR from NPs at Different GSH Concentrations. The in vitro drug release was conducted by the dialysis method. The ADR-HSA NPs solution in the membrane with concentration of $0.5 \mathrm{mg} / \mathrm{mL}$ was dialysed against $500 \mathrm{~mL} \mathrm{PBS}$ at the $\mathrm{pH} \sim 7.3$. At the predesigned time, about $0.05 \mathrm{~mL}$ ADR-HSA NPs solution was sampled and diluted by PBS to a volume of $\sim 1 \mathrm{~mL}$. At the same time, about $0.05 \mathrm{~mL}$ PBS was added to the ADR-HSA NPs solution inside membrane. For evaluating the effects of PDPH spacer on drug release, different concentrations of glutathione (GSH) were added to the drug solution. The ADR-HSA NPs solutions sampled at different time points were tested by the florescent at wavelength $\sim 480 \mathrm{~nm}$. The drug concentrations outside-/ inside-membrane were calculated and the release profile was converted.

2.8. Cellular Uptake of ADR-HSA NPs by NCI-N87 Tumor Cells. The NCI-N87 cells in logarithmic growth phase were digested by $0.25 \%$ trypsin (containing $0.05 \%$ EDTA). The cells were resuspended in DMEM containing 10\% FBS and inoculated on a glass bottomed petri dish, with $150 \mu \mathrm{L}$ per hole. The petri dish was then kept in a $5 \% \mathrm{CO}_{2}$ incubator at $37^{\circ} \mathrm{C}$ overnight. The next day, the ADR/HSA NPs and ADRHSA NPs suspension were diluted with DMEM containing $10 \%$ FBS (the concentration of ADR was $1 \mu \mathrm{M}$ ). The culture medium was sucked out of the dish; then the sample solutions were added and incubated for $2 \mathrm{~h}$ at $37^{\circ} \mathrm{C}$. The sample solution was removed, and the cells were washed using PBS solution twice and observed under laser scanning confocal microscopy (DMI4000 B, LEICA, Germany).

\subsection{The Cytotoxicity of ADR-HSA NPs to NCI-N87 Tumor} Cells. The CCK- 8 method was used to determine the in vitro growth inhibition effect of ADR/HSA NPs and ADR-HSA NPs to tumor cells. The NCI-N87 cells were digested using $0.25 \%$ trypsin, inoculated in 96 -well plates (corning, USA) with 2000 cells per hole, and cultured for $12 \mathrm{~h}$. The samples of ADR/HSA NPs and ADR-HSA NPs were diluted to $6.25 \mathrm{nM}$ to $200 \mathrm{nM}$ (ADR). The culture medium in 96-well plates was removed and the different concentrations of samples were added and incubated for $12 \mathrm{~h}$. The sample solutions were removed, and the CCK- 8 solution was added. After $10 \mathrm{~min}$, the absorbance was detected at $490 \mathrm{~nm}$ by enzyme-labeling instrument (PowerWave XS, Bio-TEK, USA). The percentage 


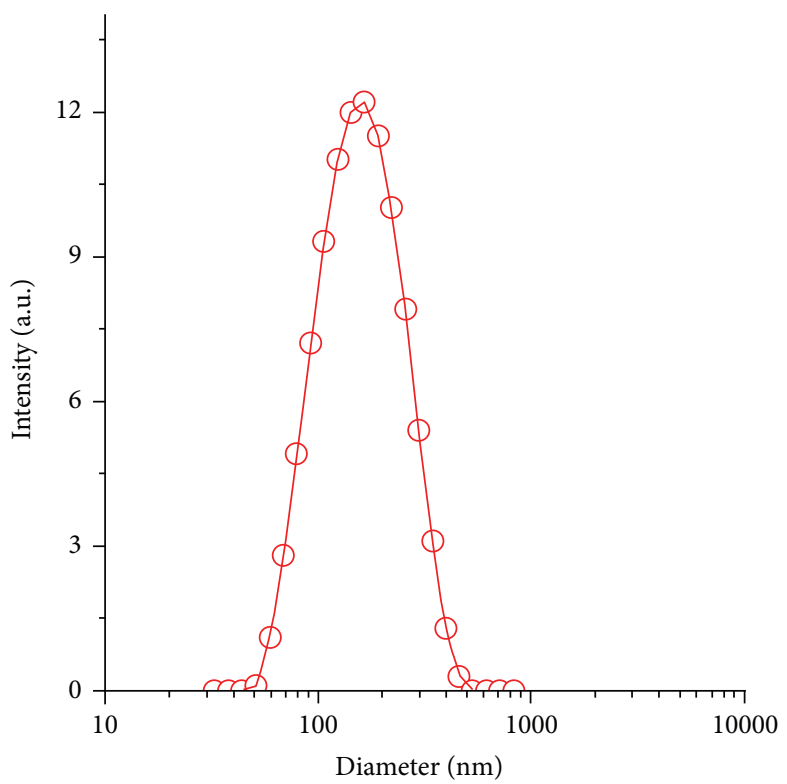

$\ominus$ HSA-ADR

(a)

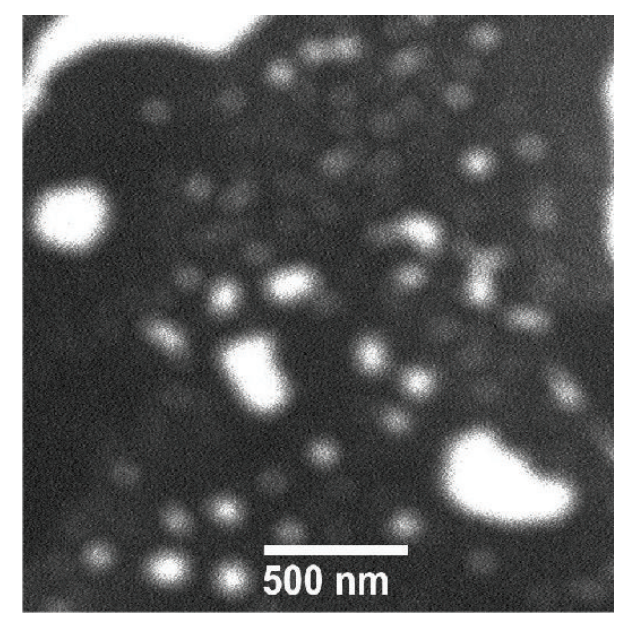

(c)

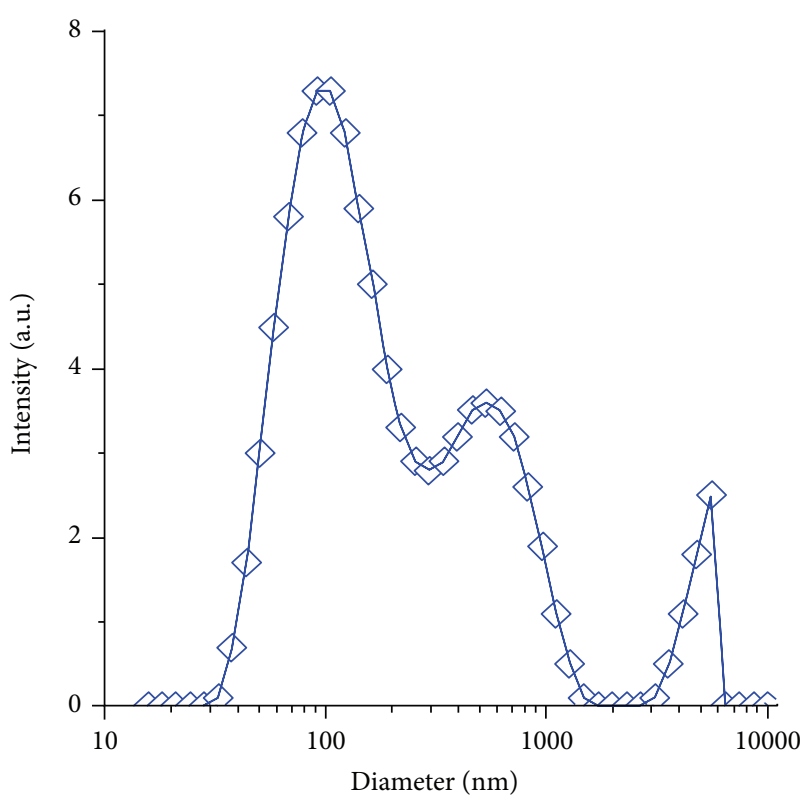

$\diamond$ HSA/ADR

(b)

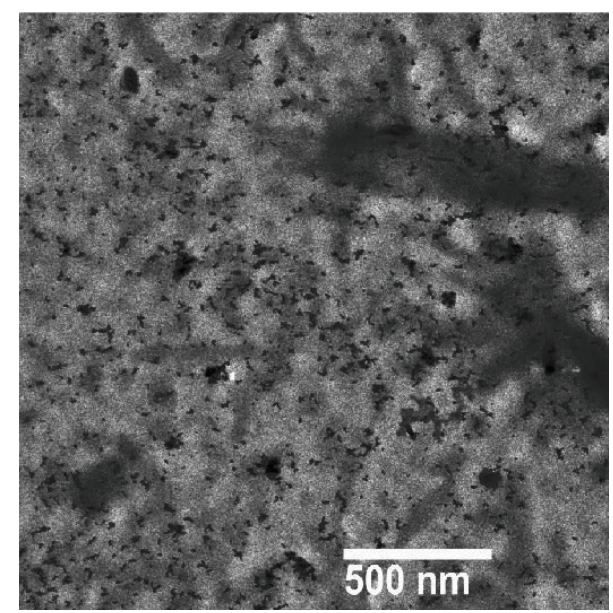

(d)

Figure 2: The size and size distribution of HSA-ADR (a), HSA/ADR (b); and the TEM photographs of HSA-ADR (c), HSA/ADR (d).

of surviving cells was calculated according to the following equation [30]:

$$
\text { Surviving cells (\%) }=\frac{\left(A_{\text {sample }}-A_{\text {blank }}\right)}{\left(A_{\text {control }}-A_{\text {blank }}\right)} \times 100,
$$

where the $A_{\text {sample }}, A_{\text {blank }}$, and $A_{\text {control }}$ are UV absorption at $485 \mathrm{~nm}$ from cell incubated with samples, the culture medium and the cell without samples.

2.10. In Vivo Tumor Accumulation Evaluation. Firstly, the $\mathrm{Balb} / \mathrm{c}$ nude mice were inoculated subcutaneously on the right back with $5 \times 10^{6}$ NCI-N87 cells (in $100 \mu \mathrm{L}$ culture medium) to develop xenografts tumor. After about 2 weeks, the volume of tumors reached about $50 \mathrm{~mm}^{3}$. For the in vivo distribution, mice bearing NCI-N87 tumor were randomly assigned to 3 groups with 3 mice/group (FICT, HSA/ADR-FITC, and HSA-ADR-FITC). Loading fluorescent isothiocyanate (FITC) HSA/ADR and HSA-ADR NPs with equivalent to $5 \mathrm{mg} / \mathrm{kg}$ dosage were administered via tail vein. 24 hours later, the tumors were viewed by IVIS Lumina II Imaging System (Xenogen), which was taken to capture the visible light photograph and luminescent image. Noted here, the mice with FITC administration at 0 hour were used as control. The in vivo images were observed with IVIS imaging system (excitation $500 \mathrm{~nm}$ ) and recorded by a built-in CCD camera [31]. For further checking the tumor accumulation, the slides of the HSA/ADR and HSA-ADR NPS groups were anaesthetized by $1.5 \%$ isoflurane in $1: 2 \mathrm{O}_{2} / \mathrm{N}_{2}$. Mice were sacrificed after $24 \mathrm{~h}$ and the heart, liver, kidney, tumor, and 
spleen were excised. These organs were also imaged with the same excitation wavelength. Then the organs were collected and immediately fixed in formalin for $1 \mathrm{~h}$. The organs were frozen in tissue-Tek-OCT and cryosections. Frozen sections were cut at $10 \mu \mathrm{m}$ and fixed with acetone at $-20^{\circ} \mathrm{C}$. After washing with PBS, sections were counterstained with $4^{\prime}, 6$ diamidino-2-phenylindole dihydrochloride (DAPI; Sigma, Fluka Chemie, Buchs, Israel) and visualized by the confocal microscopy.

2.11. The In Vivo Antitumor Effect of ADR-HSA NPs. The nude mice model was constructed by injection of NCI-N87 cells $\left(1 \times 10^{6}\right.$ cells in $200 \mu \mathrm{L}$ DMEM medium) subcutaneously. The nude mice were used for the experiment when the tumor tissue reached to $60 \mathrm{~mm}^{3}$. Three groups of nude mice models $(n=5)$ were intravenously injected with PBS (N.S.), ADR/HSA NPs, and ADR-HSA NPs, successively for 3 days. The dosage of ADR for each injection was $5 \mathrm{mg} / \mathrm{kg}$, and the total dose of ADR for each group was $300 \mathrm{mg} / \mathrm{mice}$. The volume of tumor tissue was measured every 2 days from the 1 st day to the 33 rd day. The tumor volume $(V)$ was calculated according to the following equation [32]:

$$
V_{\text {tumor }}=\frac{L W^{2}}{2},
$$

where $L$ and $W$ were the longest and shortest diameters. The related tumor volume was calculated according to the formula: related tumor volume $=$ Mean tumor volume at day recorded/mean tumor volume at day 0 .

2.12. Statistical Analysis. Student's $t$-test was performed to measure the statistical differences of different groups ( $*$ for $p<0.05 ; * *$ for $p<0.01 ; * * *$ for $p<0.001)$. The IC50 value was calculated by using the Prism ver5.02 program.

\section{Results}

3.1. Synthesis and Characterization of ADR-HSA NPs. The chemical synthesis of protein-drug conjugates was shown in Figure 1; shortly, the ADR was modified by the disulfide bond (PDPH) firstly. Then the hydrophobic chemotherapeutic drug was linked to the HSA by the PDPH. Noted here, the average number of DOX on one HAS was about 15-20 as tested by UV. The well-defined core shell micellar-like HSAADR nanoparticles were self-assembled from the amphiphilic HSA-ADR by rotary evaporation method. The particle size and size distribution of ADR/HSA NPs and ADR-HSA NPs were measured by DLS method and the results were shown in Figures 2(a) and 2(b). ADR-HSA NPs exhibited a more narrowly distributed particle size, mainly around $100 \mathrm{~nm}$ (Figure 2(a)). The size of ADR/HSA NPs exhibited a multipeak distribution, mainly centered at $1000 \mathrm{~nm}$ (Figure 2(b)). The TEM photographs of ADR-HSA NPs and ADR/HSA NPs were shown in Figures 2(c) and 2(d). The ADR-HSA NPs exhibited nearly spherical shape with uniform size similar to that obtained by DLS. But it was hard to find some uniform particles for HSA/ADR system due to the large scale and unstable structure. These results suggested the superiority of
TABLE 1: The effects of ADR, HSA/ADR, and HSA-ADR formulation composition on their in vitro/vivo performance.

\begin{tabular}{lcccc}
\hline & Size/nm & IC50/ $\mu \mathrm{M}$ & $\zeta / \mathrm{mV}$ & $\begin{array}{c}\text { Tumor inhibition } \\
\text { rate }(\%)^{*}\end{array}$ \\
\hline ADR & $0.5 \pm 0.2$ & 0.058 & - & - \\
HSA/ADR & $>1000 \pm 250$ & 0.165 & $\sim 5-10$ & 22.91 \\
HSA-ADR & $100 \pm 10$ & 0.031 & $\sim 5-10$ & 69.98 \\
\hline
\end{tabular}

${ }^{*}$ The tumor inhibition rate $=\left(V_{\text {tumor of Neg. }}-V_{\text {tumor of sample }}\right) / V_{\text {tumor of Neg. }}$.

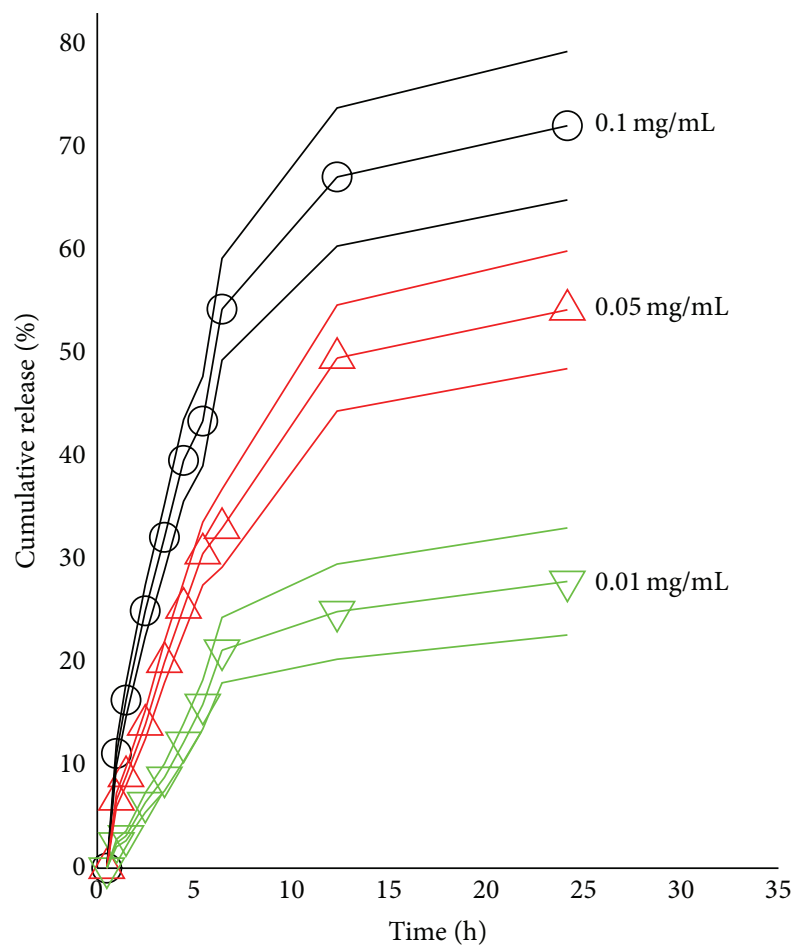

FIGURE 3: The in vitro drug release profile of HSA-ADR measured at different reducing agent GSH concentrations.

the rotary evaporation and hydration technique. By contrast, it was hard to obtain spherical dispersed particles from the TEM images of ADR/HSA NPs (Figure 2(d)). The detailed properties of the HSA/ADR and HSA-ADR were summarized in Table 1 .

3.2. In Vitro Release Profile of $A D R$. Figure 3 showed the glutathione $(\mathrm{GSH})$ concentration dependence of the drug release profile. It was found that as the GSH concentration increased from 0.01 to $0.1 \mathrm{mg} / \mathrm{mL}$, the cumulative released drug increased from $20 \%$ to $70 \%$ at the same time scale of 25 hours. There was an obvious concentration dependence of the ADR. As mentioned above, the GSH could break the PDPH linkers. So as the concentration of GSH increased, the PDPH breaking efficiency increased with more drugs released out.

3.3. Cellular Uptake of ADR-HSA NPs. Figure 4(a) showed the cellular uptake profile of the ADR as transferred by ADR, HSA/ADR, and HSA-ADR. The positive ratio of HSA/ADR 


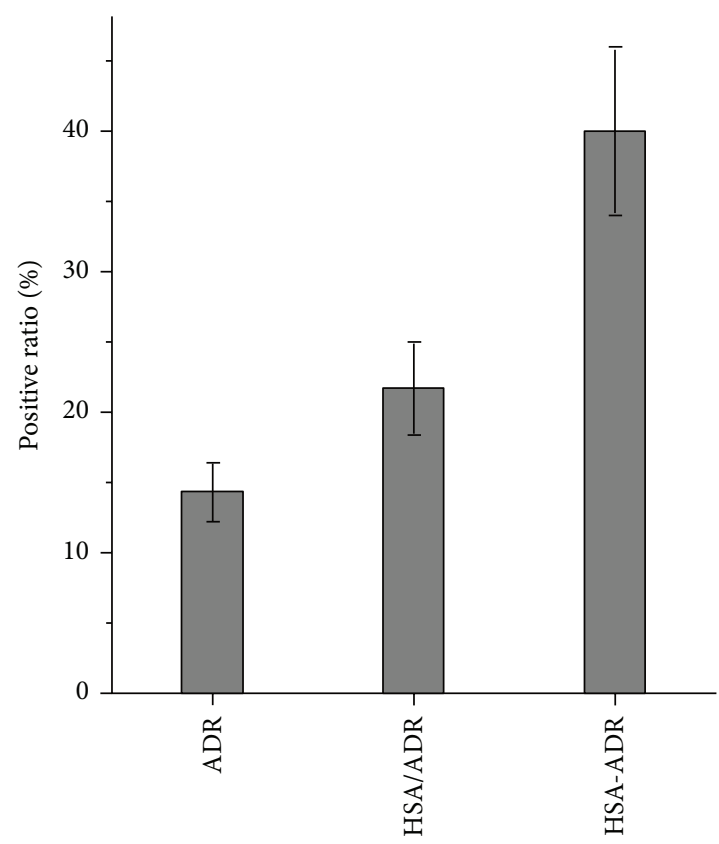

(a)
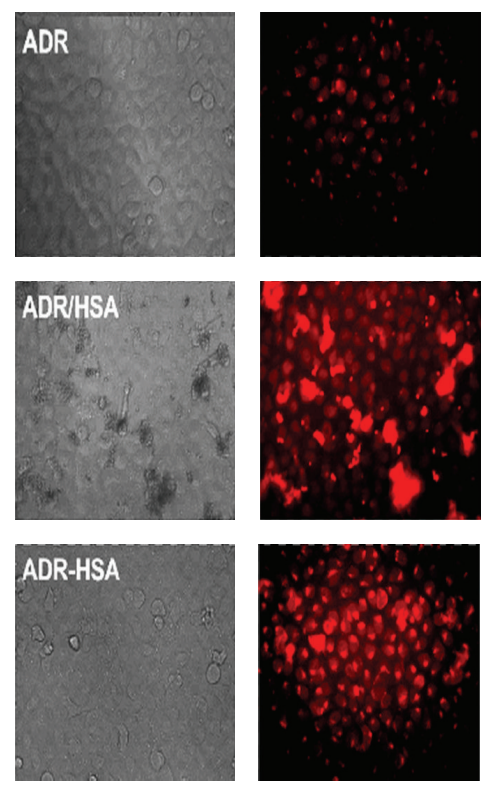

Neg

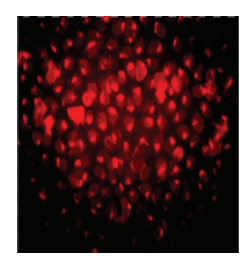

Drug

(b)
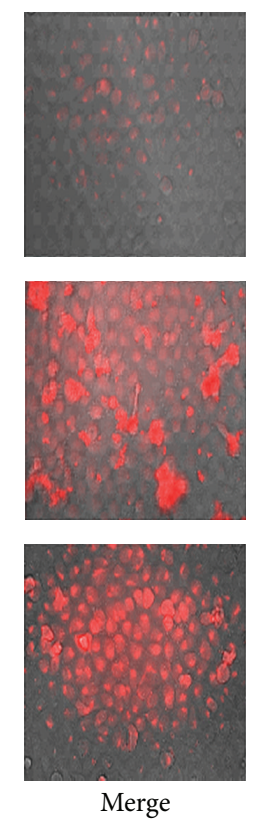

Merge

FIGURE 4: The fluorescent images of NCI-N87 tumor cells incubated with HSA, ADR, ADR/HSA NPs, and ADR-HSA NPs. The ADR/HSA NPs were aggregated and adsorbed on the bottom of petri dish, whereas the ADR-HSA NPs were internalized into the cell plasma and nucleus.

(20\%) was higher than that of ADR group (15). The positive ratio of HSA-ADR was about $40 \%$, which indicated that the cellular uptake of HSA-ADR nanoparticles was much higher than that of ADR and HSA/ADR groups. The differences of cellular uptake of ADR, ADR/HSA NPs, and ADR-HSA NPs were clearly indicated by the fluorescent images shown in Figure 4(b). The ADR can be internalized into the cell nucleus by the diffusion. For the ADR/HSA NPs group, we found some large drug-protein aggregates adsorbed on the bottom of the petri dish due to its instability, which lowered the internalization of ADR into the tumor cells. By contrast, ADR-HSA NPs can be internalized into the tumor cells with a high positive ratio. From the results, we could infer that once the ADR-HSA NPs were internalized into the tumor cell plasma, they would be degraded under the reduced circumstances, and the drug ADR would be released and entered the nucleus. Therefore, the results proved the reduction sensitivity of the ADR-HSA NPs.

3.4. In Vitro Cytotoxicity Evaluation of ADR-HSA NPs. The in vitro cytotoxicity of ADR, ADR/HSA NPs, and ADR-HSA NPs against NCI-N87 tumor cells was shown in Figure 5. At the same drug concentration, the cytotoxicity trend was HSA/ADR < ADR < HSA-ADR. The IC50 (defined as half of the cells were killed) values for ADR, ADR/HSA NPs, and ADR-HSA NPs were $0.058,0.165$, and $0.031 \mu \mathrm{M}$ as summarized in Table 1, respectively. The IC50 for ADR/HSA NPs was about 5 times higher than that for ADR-HSA NPs, indicating the significantly enhanced cytotoxicity of ADRHSA NPs to tumor cells. This result was in accord with the increased cellular uptake of ADR-HSA NPs compared with ADR/HSA NPs shown in Figure 4.

3.5. In Vivo Biodistribution and Antitumor Effect of ADRHSA NPs. Figure 6 showed the in vivo biodistribution of the HSA/ADR and HSA-ADR nanoparticles monitored by the florescent molecule FITC. From the living animal's images (Figure 6(a)), it was easy to find that the intratumor accumulation of FITC delivered by HSA-ADR conjugated system was much higher than that of HSA/ADR blend system. This enhanced tumor accumulation of FITC was further confirmed by the slide images as shown in Figure 6(b).

The results of in vivo antitumor effects of ADR/HSA NPs and ADR-HSA NPs were shown in Figure 7. Compared with N.S., ADR/HSA NPs $(p<0.01)$ and ADR-HSA NPs $(p<0.001)$ showed a significantly more enhanced antitumor effect. Furthermore, the antitumor effect of ADR-HSA NPs was significantly higher than that of ADR/HSA NPs ( $p<$ 0.001 ). The tumor inhibitory rate of ADR/HSA NPs and ADR-HSA NPs was $22.91 \%$ and $69.98 \%$, respectively. The enhanced inhibitory effect of ADR-HSA NPs should be in accord with their reduction sensitivity and the increased cellular uptake by tumor cells.

\section{Discussion}

It was known that the clinical application of small molecular chemotherapeutic drug adriamycin (ADR) was limited by its low solubility and high side effects. For overcoming the drawbacks, new nanoformulations based on liposomes, micelles were developed, among which, a successful sample 


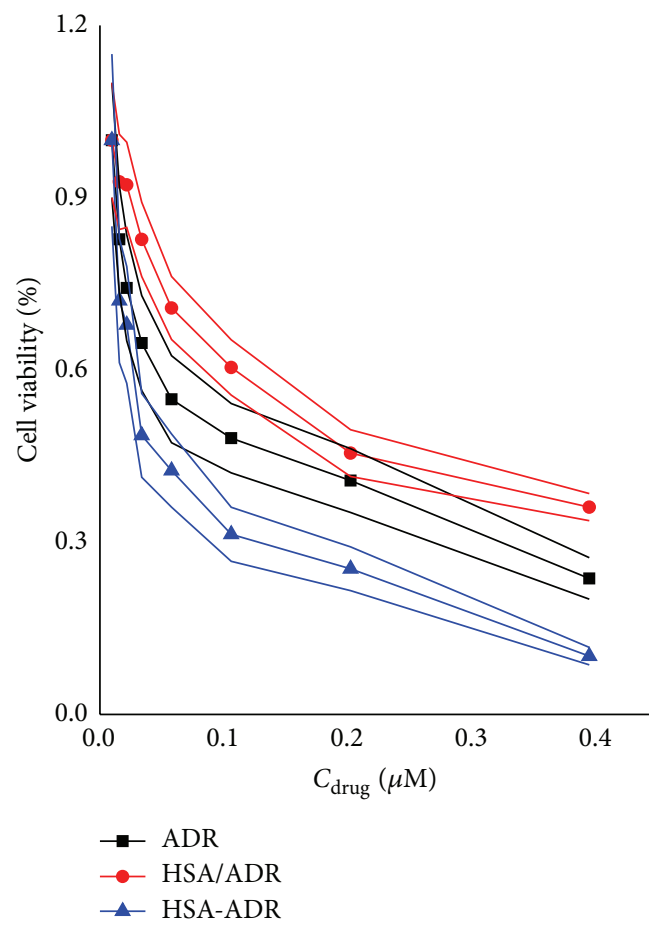

FIgURE 5: The in vitro cytotoxicity of ADR, ADR/HSA NPs, and ADR-HSA NPs to NCI-N87 tumor cells. ADR-HSA NPs showed a much lower IC50 value than ADR/HSA NPs, indicating the enhanced antitumor effect of ADR-HSA NPs compared with ADR/HSA NPs.
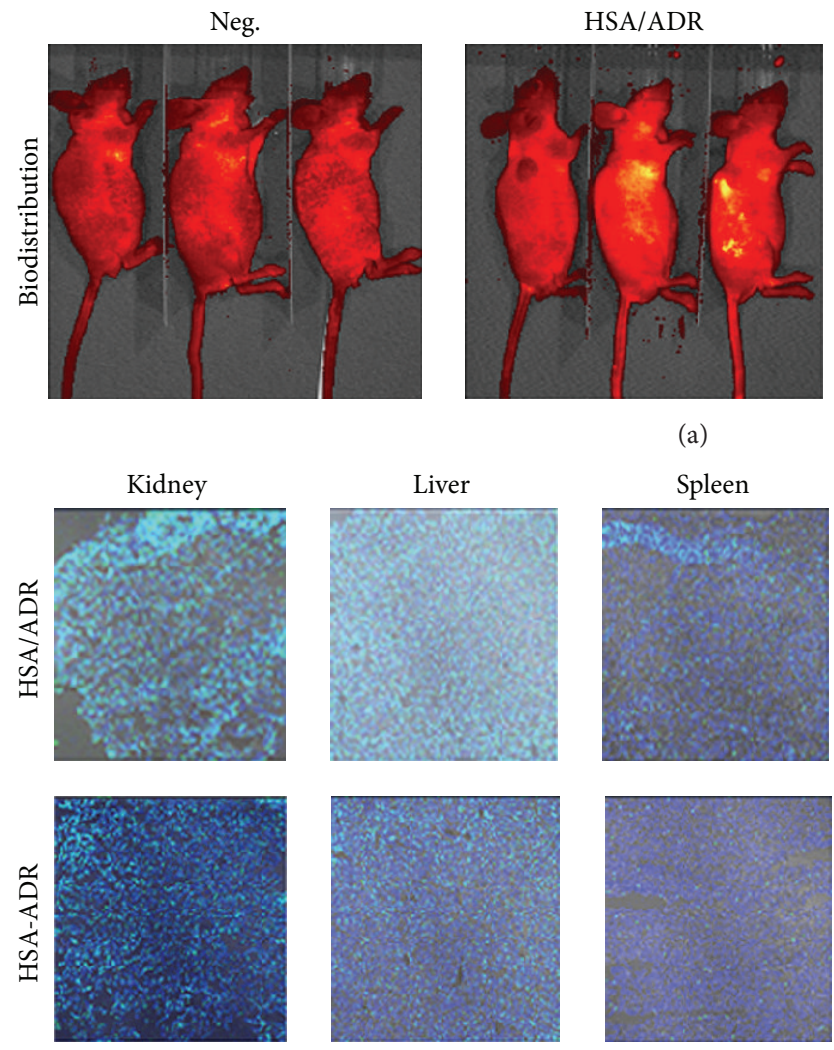

(a)
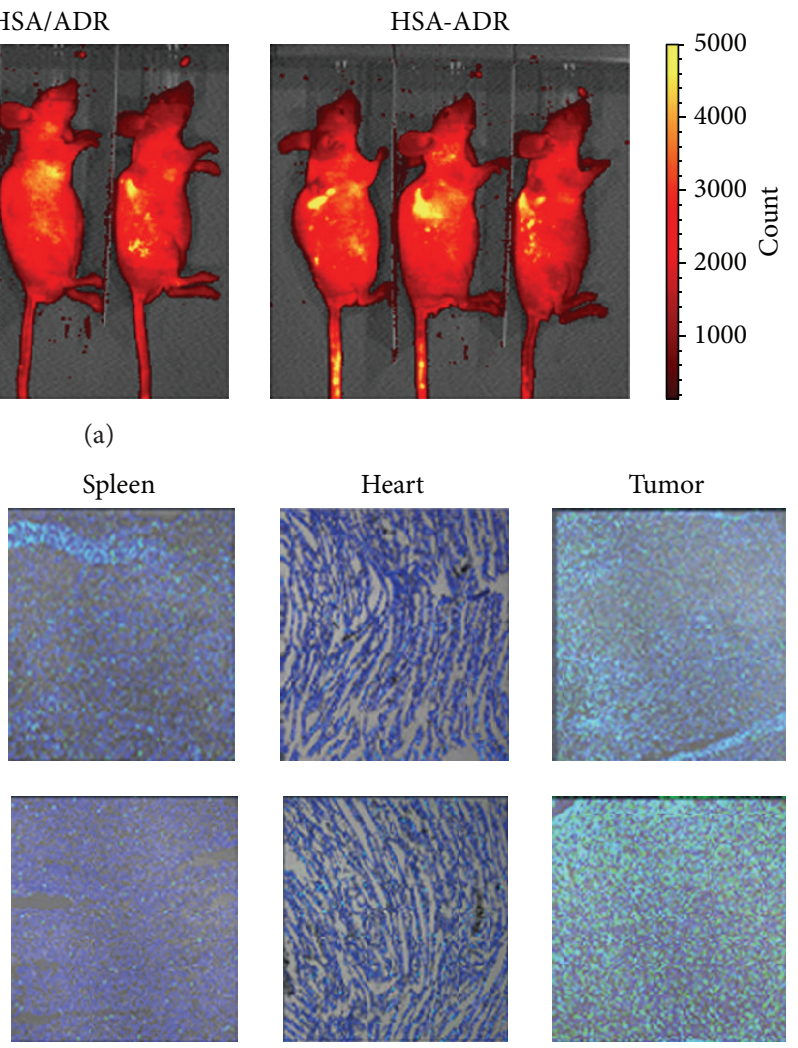

(b)

FIgURE 6: The biodistributions of Neg, ADR/HSA NPs, and ADR-HSA NPs illustrated by the living animal image (a) and the FITC accumulations in different organs' slides indicated by CLSM (b). 


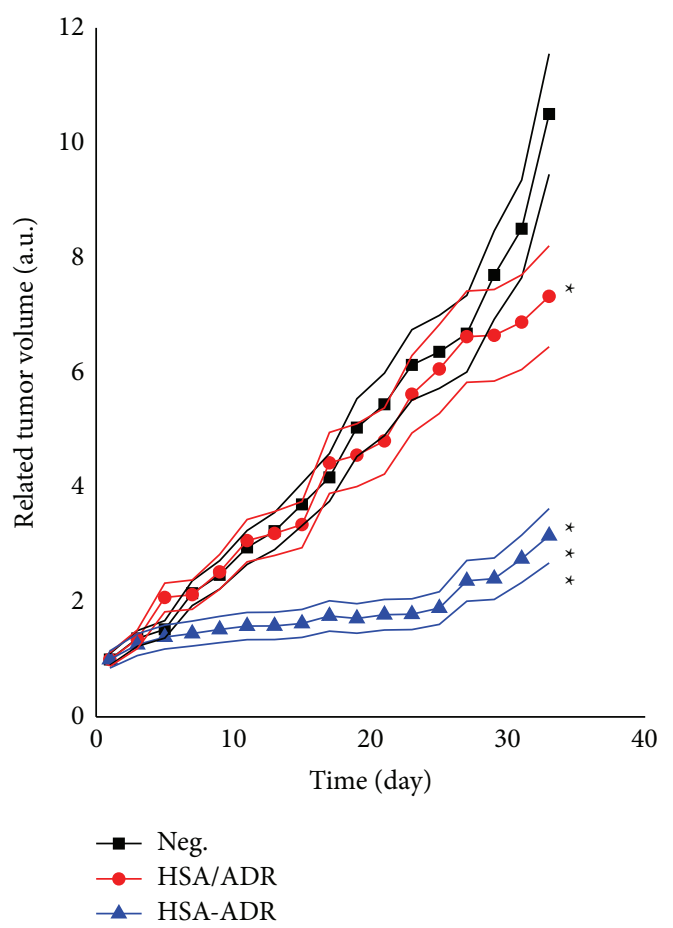

Figure 7: The inhibitory effect of N.S., ADR/HSA NPs, and ADR-HSA NPs shown as the relative tumor volume profiles. The antitumor effect of ADR-HSA NPs was much higher than that of ADR/HSA NPs.

was the HSA/PTX blend. This nanomedicine has been widely used in clinics. However, there still existed serious cytotoxicity such as bone marrow suppression, immunosuppression, and cardiac toxicity, which still strongly limited its clinic application. The limitation was attributed to the in vivo instability, drug leakage, and insufficient drug release. In this paper, we adopted a simple and feasible rotary evaporation and hydration technique to prepare novel reduction-sensitive nanoparticles ADR-HSA NPs. As evaluated by the UV and DLLS, the number of ADR conjugated on one HAS was about 15-20. As shown in Figure 1, the ADR-HSA NPs were successfully obtained by the rotary evaporation method. Its well-defined core shell structure and narrow size distribution were confirmed by the DLS and TEM as shown in Figure 2. To the best of our knowledge, this may be the first report on preparation of micellar-like HSA-ADR carriers with cleavable PDPH linkers.

In this new ADR-HSA NPs formulation, the outer shell was a biocompatible protein HSA. The inner core was composite of the hydrophobic drug ADR, while in the middle of the nanoparticles was a redox-sensitive linker PDPH, which can be degraded at a related high concentration of GSA, leading to the controlled drug release as shown in Figure 3. But it was stable when circulated in the blood due to the low GSH concentration. Additionally, compared with the HSA/ADR blends, the HSA-ADR NPs held the stable core shell structure with narrow distribution as confirmed by both Figures 1 and 2. All these properties showed that the HSAADR NPs were stable, which indicated that the ADR loaded

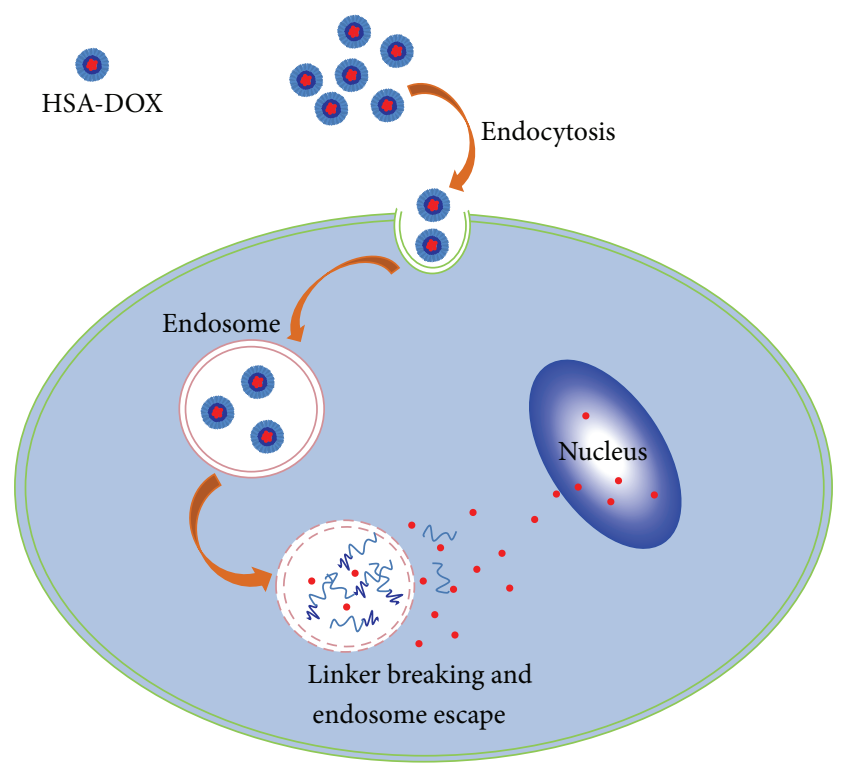

FIGURE 8: The scheme illustrated the mechanism of the cellular uptake and intracellular drug release of the HSA-ADR nanodrug delivery system.

in the micelles might not be leaked at the blood circulation. These characteristics could overcome the shortcomings like instability of traditional nanoformulation (HSA/PTX). The ADR-HSA NPs showed an increased cellular uptake with a high positive ratio shown in Figure 4 and an enhanced cytotoxicity shown in Figure 5.

The above-mentioned serum stability of the ADR-HSA NPs further enhanced the in vivo tumor accumulation as shown in Figure 6. This stable structure increased the interaction possibility of NPs with the tumor tissues [33]. The intratumor accumulation was thus obviously enhanced by the well-known EPR effects $[34,35]$. On the intracellular level, the ADR-HSA NPs uptake was mainly dominated by endocytosis to form the endosome as shown in Figure 8. Noted here, the concentration of intracellular glutathione (GSH/GSSG) was about $0.5 \sim 10 \mathrm{~mol} / \mathrm{L}$, which was over 200 times the extracellular GSH concentration $2 \sim 20 \mu \mathrm{mol} / \mathrm{L}$. The PDPH linkers were thus remarkably degraded by such a high concentration of GSH, which resulted in the ADR release. Therefore, disulfidecontained drug carriers can timely release drugs dependent on the concentration of GSH in tumor cells. So compared with the HSA/ADR system, the ADR-HSA NPs significantly enhanced tumor growth inhibition as shown in Figure 7. In this study, we proved that the ADR can be rapidly released from the ADR-HSA under high GSH, thereby increasing the inhibitory effect to gastric tumors. Therefore, the disulfidecontained polymeric prodrug and nanoparticles are potential carrier systems for the treatment of tumors.

\section{Conclusion}

In summary, by a facile chemical conjugation of chemotherapeutic drug ADR to the biocompatible protein HSA, an 
amphiphilic molecule HSA-ADR was obtained with disulfide bond PDPH. Then the novel micellar-like ADR-HSA NPs were constructed by rotary evaporation method, which exhibited a well-defined core shell structure and GSHsensitive releasing profile. The ADR release was controlled by the concentration of GSH. The in vitro cytotoxicity against NCI-N87 gastric cancer showed that such ADRHSA NPs containing redox-sensitive PDPH linkers obviously enhanced the cellular uptake of ADR. In addition, the intratumor accumulation of such ADR-HSA NPs was much higher than that of the HSA/ADR blend due to its high serum stability and tumor accumulation. Consequently, the in vivo tumor inhibition by this ADR-HSA NPs was significantly promoted as intravenous administration to the $\mathrm{Balb} / \mathrm{c}$ nude mice bearing NCI-N87 gastric tumor. These results indicated that disulfide-bond-containing ADR-HSA NPs were an effective nanodrug delivery system for targeting therapy of gastric cancer. This study may provide a new idea for the development of nanomedicine.

\section{Conflict of Interests}

The authors declare that there is no conflict of interests regarding the publication of this paper.

\section{Authors' Contribution}

Lin Chen, Feng Chen, Mengxin Zhao, Xiandi Zhu, and Changhong Ke contributed equally.

\section{Acknowledgments}

This work was financially supported by Ministry of Science and Technology of China (2012CB934002, 2012AA02A304), the National Natural Science Foundation of China including the project (81171450, 31470964, and 81301537), and Shanghai Key Special Medical Project (ZK2012A26, PWZxq2014-04).

\section{References}

[1] C. E. Myers, W. P. McGuire, R. H. Liss, I. Ifrim, K. Grotzinger, and R. C. Young, "Adriamycin: the role of lipid peroxidation in cardiac toxicity and tumor response," Science, vol. 197, no. 4299, pp. 165-167, 1977.

[2] W. Li, J. Li, J. Gao et al., "The fine-tuning of thermosensitive and degradable polymer micelles for enhancing intracellular uptake and drug release in tumors," Biomaterials, vol. 32 , no. 15, pp. 3832-3844, 2011.

[3] G. Joshi, R. Sultana, J. Tangpong et al., "Free radical mediated oxidative stress and toxic side effects in brain induced by the anti cancer drug adriamycin: insight into chemobrain," Free Radical Research, vol. 39, no. 11, pp. 1147-1154, 2005.

[4] H.-C. Yen, T. D. Oberley, S. Vichitbandha, Y.-S. Ho, and D. K. St. Clair, "The protective role of manganese superoxide dismutase against adriamycin-induced acute cardiac toxicity in transgenic mice," The Journal of Clinical Investigation, vol. 98, no. 5, pp. 1253-1260, 1996.

[5] K. A. Janes, M. P. Fresneau, A. Marazuela, A. Fabra, and M. J. Alonso, "Chitosan nanoparticles as delivery systems for doxorubicin," Journal of Controlled Release, vol. 73, no. 2-3, pp. 255-267, 2001.

[6] J.-Z. Du, X.-J. Du, C.-Q. Mao, and J. Wang, “Tailor-Made dual $\mathrm{pH}$-sensitive polymer-doxorubicin nanoparticles for efficient anticancer drug delivery," Journal of the American Chemical Society, vol. 133, no. 44, pp. 17560-17563, 2011.

[7] S. Dreis, F. Rothweiler, M. Michaelis, J. Cinatl Jr., J. Kreuter, and K. Langer, "Preparation, characterisation and maintenance of drug efficacy of doxorubicin-loaded human serum albumin (HSA) nanoparticles," International Journal of Pharmaceutics, vol. 341, no. 1-2, pp. 207-214, 2007.

[8] K. Langer, S. Balthasar, V. Vogel, N. Dinauer, H. von Briesen, and D. Schubert, "Optimization of the preparation process for human serum albumin (HSA) nanoparticles," International Journal of Pharmaceutics, vol. 257, no. 1-2, pp. 169-180, 2003.

[9] M. J. Hawkins, P. Soon-Shiong, and N. Desai, "Protein nanoparticles as drug carriers in clinical medicine," Advanced Drug Delivery Reviews, vol. 60, no. 8, pp. 876-885, 2008.

[10] W. Li, S. Feng, and Y. Guo, "Tailoring polymeric micelles to optimize delivery to solid tumors," Nanomedicine, vol. 7, no. 8, pp. 1235-1252, 2012.

[11] S. Cecco, M. Aliberti, P. Baldo, E. Giacomin, and R. Leone, "Safety and efficacy evaluation of albumin-bound paclitaxel," Expert Opinion on Drug Safety, vol. 13, no. 4, pp. 511-520, 2014.

[12] F. Kratz, "Albumin as a drug carrier: design of prodrugs, drug conjugates and nanoparticles," Journal of Controlled Release, vol. 132, no. 3, pp. 171-183, 2008.

[13] K. Wosikowski, E. Biedermann, B. Rattel et al., "In vitro and in vivo antitumor activity of methotrexate conjugated to human serum albumin in human cancer cells," Clinical Cancer Research, vol. 9, no. 5, pp. 1917-1926, 2003.

[14] K. M. Osadnik and K. Jelonek, "The influence of conjugates on their functionality in the therapy of solid tumors," Acta Poloniae Pharmaceutica, vol. 70, no. 3, pp. 379-386, 2013.

[15] W. Li, H.-F. Wei, H. Li, J. Gao, S.-S. Feng, and Y.-J. Guo, "Cancer nanoimmunotherapy using advanced pharmaceutical nanotechnology," Nanomedicine, vol. 9, no. 16, pp. 2587-2606, 2014.

[16] R. Lehner, X. Wang, M. Wolf, and P. Hunziker, "Designing switchable nanosystems for medical application," Journal of Controlled Release, vol. 161, no. 2, pp. 307-316, 2012.

[17] P. Kuppusamy, H. Li, G. Ilangovan et al., "Noninvasive imaging of tumor redox status and its modification by tissue glutathione levels," Cancer Research, vol. 62, no. 1, pp. 307-312, 2002.

[18] F. H. Meng, W. E. Hennink, and Z. Y. Zhong, "Reductionsensitive polymers and bioconjugates for biomedical applications," Biomaterials, vol. 30, no. 12, pp. 2180-2198, 2009.

[19] P. Kuppusamy, M. Afeworki, R. A. Shankar et al., "In vivo electron paramagnetic resonance imaging of tumor heterogeneity and oxygenation in a murine model," Cancer Research, vol. 58, no. 7, pp. 1562-1568, 1998.

[20] C. Cui, Y.-N. Xue, M. Wu et al., "Cellular uptake, intracellular trafficking, and antitumor efficacy of doxorubicin-loaded reduction-sensitive micelles," Biomaterials, vol. 34, no. 15, pp. 3858-3869, 2013.

[21] H. He, H. Kuang, L. Yan et al., "A reduction-sensitive carrier system using mesoporous silica nanospheres with biodegradable polyester as caps," Physical Chemistry Chemical Physics, vol. 15, no. 34, pp. 14210-14218, 2013.

[22] A. Russo, W. DeGraff, N. Friedman, and J. B. Mitchell, "Selective modulation of glutathione levels in human normal versus 
tumor cells and subsequent differential responses to chemotherapy drugs," Cancer Research, vol. 46, no. 6, pp. 2845-2848, 1986.

[23] S. Bauhuber, C. Hozsa, M. Breunig, and A. Göpferich, "Delivery of nucleic acids via disulfide-based carrier systems," Advanced Materials, vol. 21, no. 32-33, pp. 3286-3306, 2009.

[24] L. P. Lv, J. P. Xu, X. S. Liu, G. Y. Liu, X. Yang, and J. Ji, “Disulfidecrosslinked biomimetic micelles: formation, thiol reactivity and cytotoxicity behavior," Macromolecular Chemistry and Physics, vol. 211, no. 21, pp. 2292-2300, 2010.

[25] H. Sun, F. Meng, R. Cheng, C. Deng, and Z. Zhong, "Reductionsensitive degradable micellar nanoparticles as smart and intuitive delivery systems for cancer chemotherapy," Expert Opinion on Drug Delivery, vol. 10, no. 8, pp. 1109-1122, 2013.

[26] C. Cui, Y.-N. Xue, M. Wu et al., "Poly(L-aspartamide)-based reduction-sensitive micelles as nanocarriers to improve doxorubicin content in cell nuclei and to enhance antitumor activity," Macromolecular Bioscience, vol. 13, no. 8, pp. 10361047, 2013.

[27] Y. Gao, L. Chen, Z. Zhang, Y. Chen, and Y. Li, "Reversal of multidrug resistance by reduction-sensitive linear cationic click polymer/iMDR1-pDNA complex nanoparticles," Biomaterials, vol. 32, no. 6, pp. 1738-1747, 2011.

[28] G. Cheng, Y. He, L. Xie et al., "Development of a reductionsensitive diselenide-conjugated oligoethylenimine nano particulate system as a gene carrier," International Journal of Nanomedicine, vol. 7, pp. 3991-4006, 2012.

[29] L. Zhang, H. Xue, Z. Cao, A. Keefe, J. Wang, and S. Jiang, "Multifunctional and degradable zwitterionic nanogels for targeted delivery, enhanced MR imaging, reduction-sensitive drug release, and renal clearance," Biomaterials, vol. 32, no. 20, pp. 4604-4608, 2011.

[30] W. Li, S. Feng, and Y. Guo, "Block copolymer for nanomedicine," Nanomedicine, vol. 7, no. 3, pp. 383-392, 2012.

[31] W. Li, H. Li, J. Li et al., "Self-assembled supramolecular nano vesicles for safe and highly efficient gene delivery to solid tumors," International Journal of Nanomedicine, vol. 7, pp. 46614677, 2012.

[32] W. Li, H. Zhao, W. Qian et al., "Chemotherapy for gastric cancer by finely tailoring anti-Her2 anchored dual targeting immunomicelles," Biomaterials, vol. 33, no. 21, pp. 5349-5362, 2012.

[33] E. Ayano, M. Karaki, T. Ishihara, H. Kanazawa, and T. Okano, "Poly (N-isopropylacrylamide)-PLA and PLA blend nanoparticles for temperature-controllable drug release and intracellular uptake," Colloids and Surfaces B: Biointerfaces, vol. 99, pp. 67-73, 2012.

[34] W. Li, L. Zhang, G. Zhang et al., "The finely regulating well-defined functional polymeric nanocarriers for anti-tumor immunotherapy," Mini-Reviews in Medicinal Chemistry, vol. 13, no. 5, pp. 643-652, 2013.

[35] W. Li, M. Nakayama, J. Akimoto, and T. Okano, "Effect of block compositions of amphiphilic block copolymers on the physicochemical properties of polymeric micelles," Polymer, vol. 52, no. 17, pp. 3783-3790, 2011. 

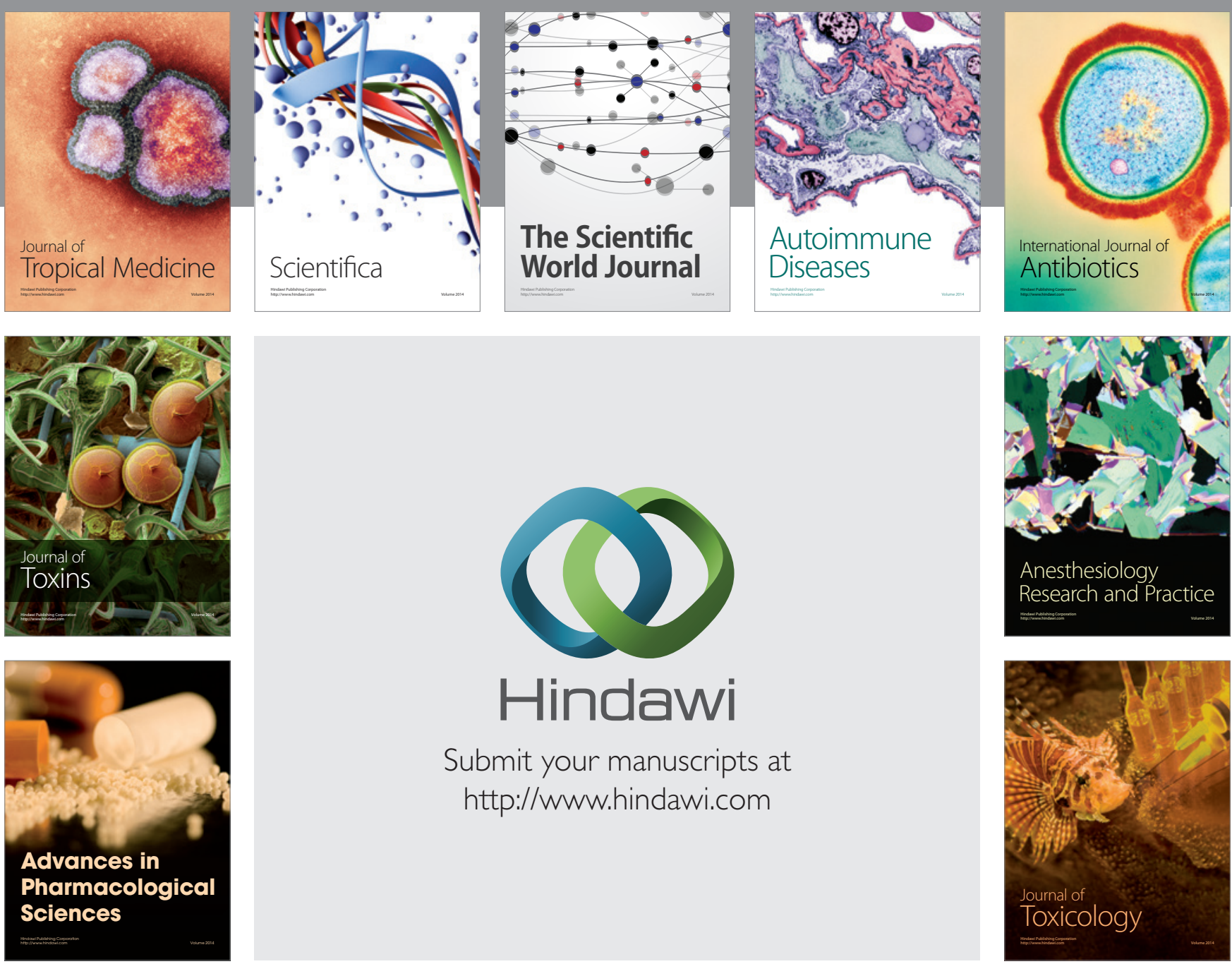

\section{Hindawi}

Submit your manuscripts at

http://www.hindawi.com
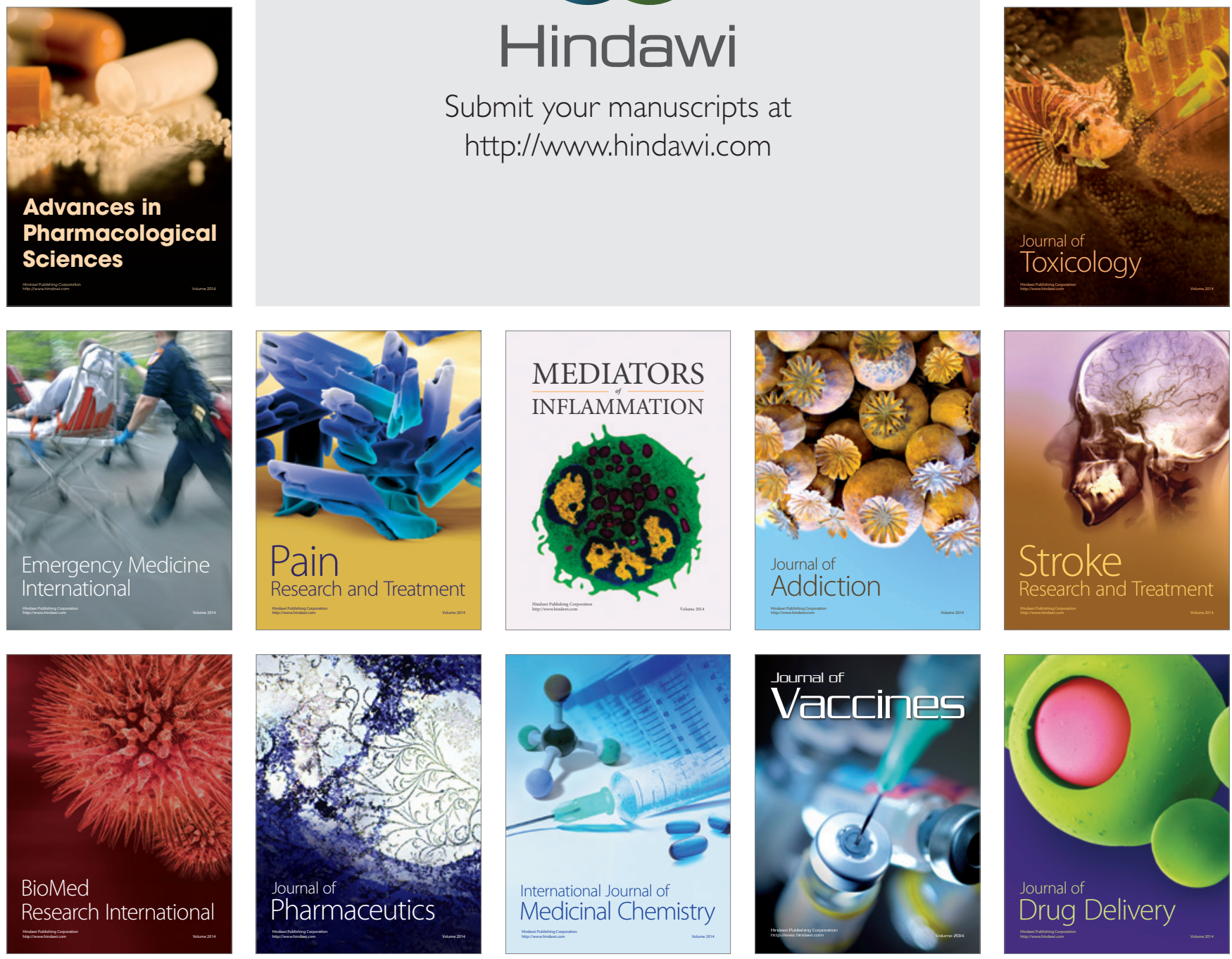